\title{
Sintering Bonding Process with Ag Nanoparticle Paste and Joint Properties in High Temperature Environment
}

\author{
Jianfeng Yan, ${ }^{1,2}$ Dongyue Zhang, ${ }^{1}$ Guisheng Zou, ${ }^{1}$ Lei Liu, ${ }^{1}$ Hailin Bai, \\ Aiping $\mathrm{Wu},{ }^{1}$ and $\mathrm{Y}$. Norman $\mathrm{Zhou}^{1,3}$ \\ ${ }^{1}$ Department of Mechanical Engineering, Tsinghua University, Beijing 100084, China \\ ${ }^{2}$ Department of Aerospace and Mechanical Engineering, University of Southern California, Los Angeles, CA 90089, USA \\ ${ }^{3}$ Department of Mechanical and Mechatronics Engineering, University of Waterloo, 200 University Avenue West, \\ Waterloo, ON, Canada N2L $3 G 1$
}

Correspondence should be addressed to Jianfeng Yan; yanjf@tsinghua.org.cn

Received 4 November 2015; Revised 15 December 2015; Accepted 31 January 2016

Academic Editor: Jinting Jiu

Copyright (C) 2016 Jianfeng Yan et al. This is an open access article distributed under the Creative Commons Attribution License, which permits unrestricted use, distribution, and reproduction in any medium, provided the original work is properly cited.

\begin{abstract}
Ag nanoparticle paste is prepared based on the polyol method and subsequent concentration by centrifuging. The sintering bonding process using Ag nanoparticle paste at different bonding pressures is studied. The joint strengths are increased as the bonding pressure increases from $0 \mathrm{MPa}$ to $7.5 \mathrm{MPa}$. This is due to the fact that the higher assistant bonding pressure is beneficial to the growth of neck size between the adjacent particles and forms denser sintered Ag layers. The joint strength bonded under $10 \mathrm{MPa}$ is lower than that bonded under 7.5 MPa, which may be due to the residue of organic component in the sintered Ag layer. The joint properties bonded with $\mathrm{Ag}$ nanoparticle paste in high temperature environment are evaluated by heat treatments at temperatures ranges of $200-350^{\circ} \mathrm{C}$ for 50 hours. The results show that the mechanical properties of joint with Ag nanoparticle paste are better than the joint with $\mathrm{Pb} 95 \mathrm{Sn} 5$ solder after storage at high temperatures.
\end{abstract}

\section{Introduction}

Electronic packaging is one of the key technologies during electronic product manufacturing. Electronic packaging materials provide the medium for electronic interconnections and mechanical support between the chips and the bonding pads. Lead-based solders are used widely as electronic packaging materials. However, $\mathrm{Pb}$ and its compounds pose serious threat to human health and environment when they are disposed. The bonding material without $\mathrm{Pb}$ is necessary to protect the environment and human health [1].

The emergence of nanometer technology gives a possible solution for these problems. When particles are reduced to the nanoscale, some special properties are obvious, such as increase of the diffusion coefficient and decrease of the melting point [2-5]. Ag has high electrical and thermal conductivity and exhibits good fatigue performance. These good properties, as well as its high melting point $\left(960^{\circ} \mathrm{C}\right)$, make Ag a suitable material for high temperature packaging applications. Silver nanoparticles can be sintered at low temperature and have the potential to be a $\mathrm{Pb}$-free, highperformance interconnecting material for high temperature electronic applications [6].

Recently, there has been an increasing interest in developing the sintering bonding process using metal nanoparticle paste [7-12]. Typically, the organic component was added to the silver nanoparticles to prevent the self-cohesion of metal nanoparticles [9, 13-16]. However, the added organic component has detrimental effects in the sintering bonding applications due to its residue in the joint layer [17]. The reduction of organic components is beneficial to form joints with lower bonding temperatures and assistant bonding pressures [18]. A method to prepare Ag nanoparticle paste based on the polyol method was developed to prepare $\mathrm{Ag}$ nanoparticle paste with a high Ag content of 96.1 mass\% $[18,19]$. It has been demonstrated that the joint can be formed 
without using assistant bonding pressure [18]. The sintering mechanisms and joint strength related with the microstructure have been reported [20]. In this study, the bonding processes under different assistant bonding pressures using Ag nanoparticle paste are studied. The joint properties in high temperature environment are evaluated by the storage at high temperatures. The good service performance of joint bonded with $\mathrm{Ag}$ nanoparticle paste is demonstrated by this experiment.

\section{Experimental Procedure}

The Ag nanoparticle paste used in this study was prepared by the chemical reduction method and subsequent concentration without further addition of organic component [18]. Firstly, silver nanoparticle solution was synthesized based on the modified polyol method [21]. The $\mathrm{AgNO}_{3}$ was reduced in EG solution, and polyvinylpyrrolidone (PVP) was used as a protecting agent to prevent it from aggregation. The polyol in the polyol process acted as both a solvent and a reducing agent. This Ag particle paste was prepared by condensing Ag nanoparticle solution with a centrifuge at $7000 \mathrm{rpm}$ for 20 min without addition of organic component. The bonding process using Ag nanoparticle paste was conducted in an oven at $250^{\circ} \mathrm{C}$ with bonding pressures from $0 \mathrm{MPa}$ to $10 \mathrm{MPa}$ in air and a holding time of $30 \mathrm{~min}$. The bonding specimens were $\mathrm{Ni} / \mathrm{Ag}$ coated $\mathrm{Cu}$ discs as shown in Figure 1. The nickel plating followed by silver plating was applied using electrolysis to protect the $\mathrm{Cu}$ discs from oxidation. These $\mathrm{Ni} / \mathrm{Ag}$ coated $\mathrm{Cu}$ discs were bonded using Ag nanoparticle paste. The Ag nanoparticles were applied on the faying surfaces of $\Phi 6 \mathrm{~mm} \mathrm{Cu}$ disc, which was set on the $\Phi 10 \mathrm{~mm}$ $\mathrm{Cu}$ disc.

The synthesized Ag nanoparticles were characterized by scanning electron microscopy (SEM) and transmission electron microscopy (TEM). Ultraviolet-visible (UV-vis) spectroscopy was conducted during the course of the reaction using a quartz cuvette by a UV-vis spectrophometer. The bonding strength was measured as shear strength using the Thermal-Mechanical Simulator Gleeble 1500D with a displacement speed of $5 \mathrm{~mm} / \mathrm{min}$. To evaluate the joint properties the high environments, the joints bonded using Ag nanoparticle paste were heat treated at different temperatures from $200^{\circ} \mathrm{C}$ to $350^{\circ} \mathrm{C}$ for 50 hours. The joints bonded using typical high-melting-point solder Pb95Sn5 were also prepared and heat treated at the same parameters for comparison. After the heat treatment, the joints using both Ag nanopartcle paste and solder Pb95Sn5 were tested using the Thermal-Mechanical Simulator Gleeble 1500D with a displacement speed of $5 \mathrm{~mm} / \mathrm{min}$ under room temperature. Three samples were tested for the same bonding parameters.

\section{Results and Discussion}

3.1. Characteristics of the Synthesized Ag NP Paste. Due to Ag nanoparticles with different shapes exhibiting surface plasmon resonance (SPR) bands at different frequencies, the

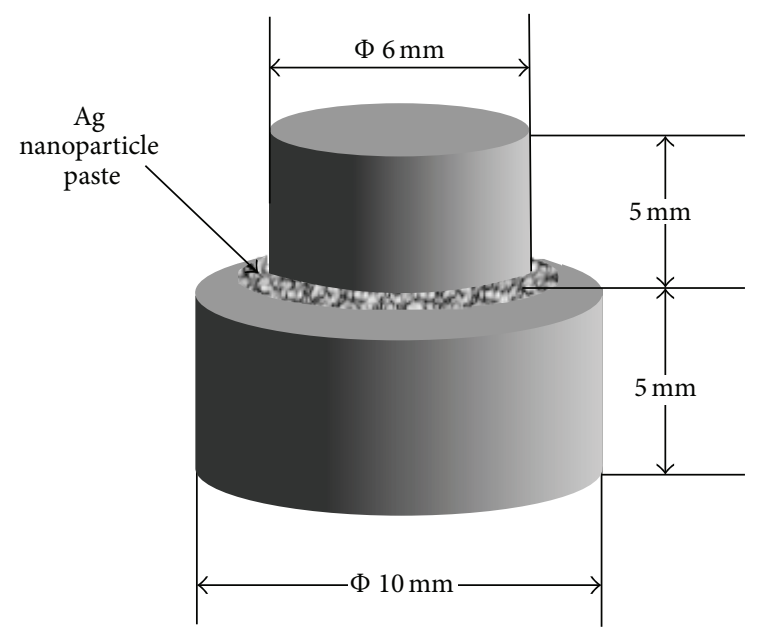

FIGURE 1: Schematic illustration of the bonding specimens of big $\mathrm{Cu}$ $\operatorname{disc}(\Phi=10 \mathrm{~mm}, h=5 \mathrm{~mm})$ and small Cu disc $(\Phi=6 \mathrm{~mm}, h=$ $5 \mathrm{~mm})$.

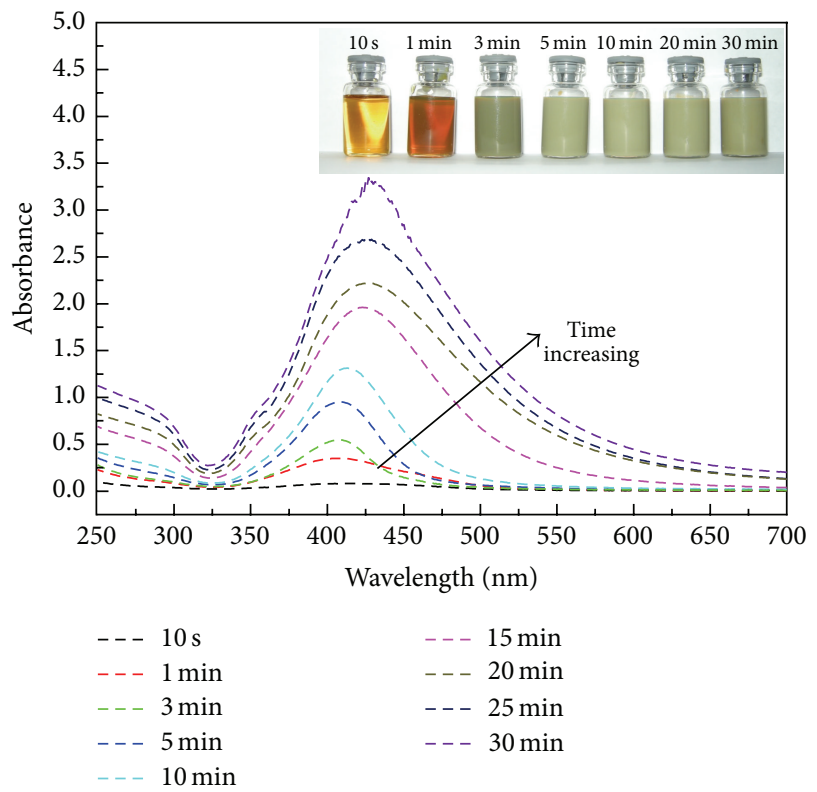

FIGURE 2: UV-vis absorption spectral evolution during the formation of Ag nanoparticles.

UV-vis spectroscopic method can be used to track the morphological evolution involved in the growth process of $\mathrm{Ag}$ nanoparticles [22]. It is reported that reduction mechanism of Ag nanoparticles by ethylene glycol (EG) likely involves the following chemical reactions [23]:

$$
\begin{aligned}
& 2 \mathrm{HOCH}_{2}-\mathrm{CH}_{2} \mathrm{OH}=2 \mathrm{CH}_{3} \mathrm{CHO}+2 \mathrm{H}_{2} \mathrm{O} \\
& 2 \mathrm{CH}_{3} \mathrm{CHO}+2 \mathrm{AgNO}_{3} \\
& \quad=\mathrm{CH}_{3} \mathrm{CO}-\mathrm{COCH}_{3}+2 \mathrm{Ag}+2 \mathrm{HNO}_{3}
\end{aligned}
$$

Figure 2 shows the UV-visible spectra obtained from the solutions sampled at different reaction times. The Ag nanoparticles are generated gradually, which is clearly indicated by 


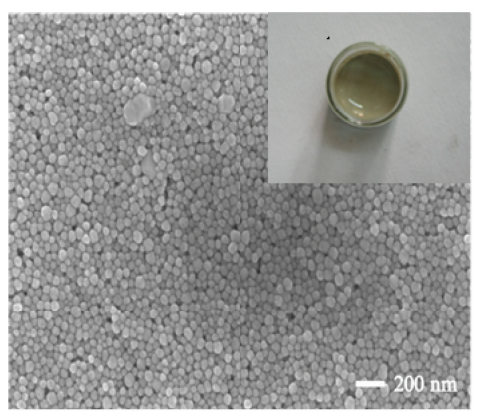

(a)

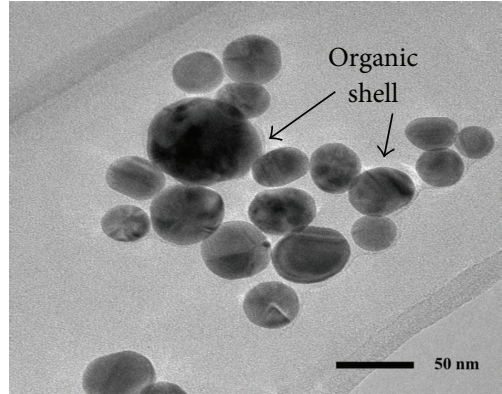

(b)

Figure 3: SEM (a) and TEM (b) image of Ag nanoparticles (inset showing the photo of Ag nanoparticle paste contained in a glass bottle).

the increasing intensity of the surface plasmon band in the $\mathrm{UV}$-vis spectra. No peak is detected from the solution sample at $10 \mathrm{~s}$, indicating that almost no Ag nanoparticles were formed by this time. The appearance of a weak plasmon peak at about $402 \mathrm{~nm}$ (reaction time $1 \mathrm{~min}$ ) indicated the formation of $\mathrm{Ag}$ colloids. In the period from 3 to $10 \mathrm{~min}$, the intensity of the peak increased and the peak position shifted from 407 to $428 \mathrm{~nm}$. This increase of intensity and red-shift in energy indicates the change of both number and size of $\mathrm{Ag}$ nanoparticles. As the reaction proceeded from 10 to $15 \mathrm{~min}$, there is an obvious increase for peak position and intensity. This suggests the generation and growth of Ag nanoparticles. However after $15 \mathrm{~min}$, the change of peak position is not obvious, although a significant increase in intensity is observed. This indicates that the size or shape of initially formed Ag nanoparticles is basically kept constant from 15 to $30 \mathrm{~min}$ and new Ag nanoparticles continue to form.

Figure 3 shows the SEM and TEM images of Ag nanoparticles after the reaction [20]. The Ag nanoparticles are mainly spherical with diameters of about $40 \mathrm{~nm}$. In the TEM image the organic shell coated on the Ag particle surface can be observed. It is shown that this organic shell can prevent the aggregation of the particles before the bonding process [18].

3.2. Effect of Bonding Pressures on the Joint Strength and Microstructure. The sintering bonding processes at different bonding pressures from $0 \mathrm{MPa}$ to $10 \mathrm{MPa}$ at $250^{\circ} \mathrm{C}$ were performed. Figure 4 illustrates the shear strength of joints bonded using Ag nanoparticle paste at different bonding pressures. It can be seen that a joint with shear strengths higher than $15 \mathrm{MPa}$ is formed without the assistant bonding pressure. This is attributed to the decrease of organic content in the Ag nanoparticle paste [18]. The decrease of organic content benefits the formation of joints at low temperature and low assistant bonding pressures. With the increase of bonding pressures from $2 \mathrm{MPa}$ to $7.5 \mathrm{MPa}$, the joint strength increased gradually. The joint bonded at $7.5 \mathrm{MPa}$ has a high strength of about $60 \mathrm{MPa}$. However, with the further increase of the bonding pressure to $10 \mathrm{MPa}$, the joint strength is decreased to $55 \mathrm{MPa}$. Figure 5 shows the optical and SEM top fracture surfaces of the joints bonded at different bonding pressures. It can be seen that the joint formed without assistance pressure



FIGURE 4: Shear strength of joints bonded using Ag nanoparticle paste at different bonding pressures.

shows less fracture traces. This suggests that during the bonding process without pressure the partial particles form interconnections between the adjacent nanoparticles. For the joints formed at pressures from $2 \mathrm{MPa}$ to $10 \mathrm{MPa}$, obvious fracture traces can be observed, which correspond to the high joint strength. The effect of bonding pressures can be explained by the sphere-to-sphere model [20]. It has been demonstrated that the joint strength is proportional to the square of the neck size ratio of sintered Ag nanoparticles in the sphere-to-sphere model. The pressure between the two adjacent nanoparticles in the sintered layer will increase with higher assistant pressure according to Herring's theorem [24]. The higher pressure between the two adjacent nanoparticles is beneficial to the growth of neck size during the sintering bonding process. Since the assistant bonding pressure will increase the neck size during the sintering bonding process of Ag nanoparticles, it is reasonable to expect that joint strength increases at the higher bonding pressure. The bonding pressure will also play a role in increasing the density of sintered Ag nanoparticles, which will increase the effective bonding area in the joints. It should be noted that the joint strength formed at $10 \mathrm{MPa}$ is lower than that formed at 7.5 MPa. This 


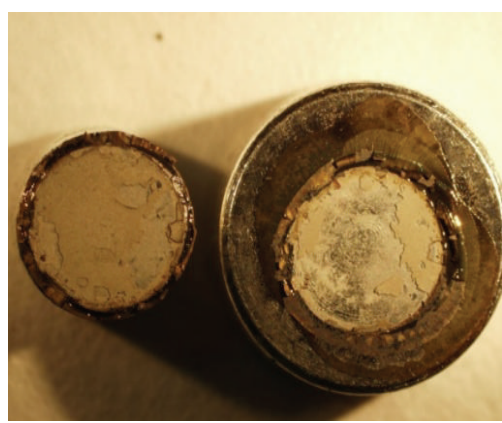

(a)



(d)

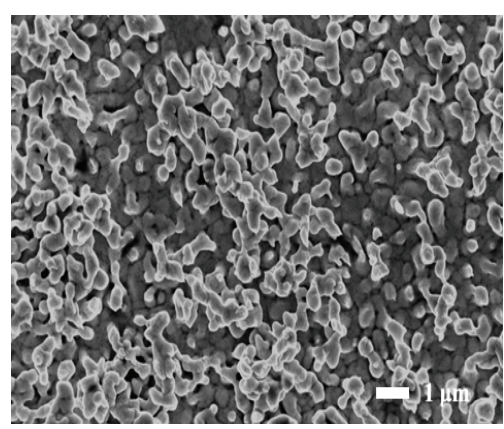

(b)

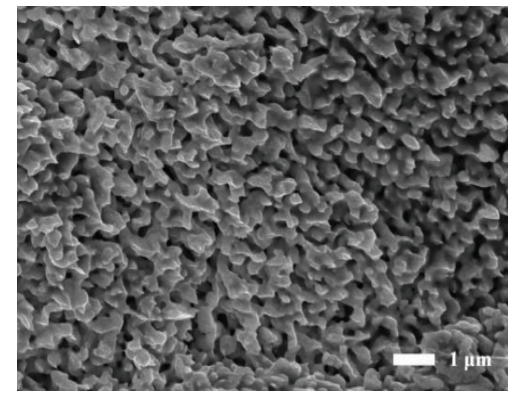

(e)

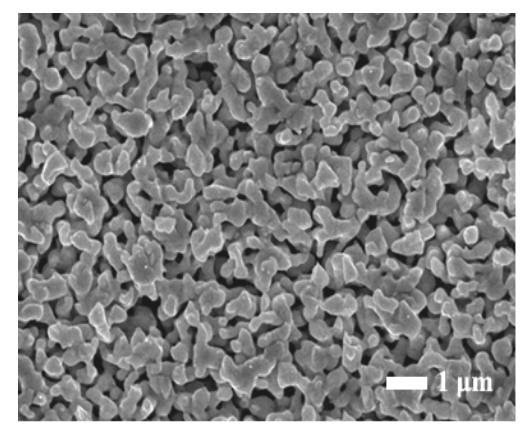

(c)

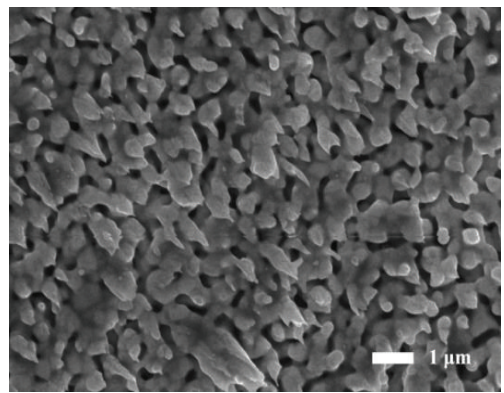

(f)

FIGURE 5: Optical and SEM top fracture surfaces of the joints bonded at different pressures: optical microscope at low magnification (a); $0 \mathrm{MPa}$ (b); $2 \mathrm{MPa}$ (c); $5 \mathrm{MPa}$ (d); $7.5 \mathrm{MPa}$ (e); $10 \mathrm{MPa}$ (f).



(a)

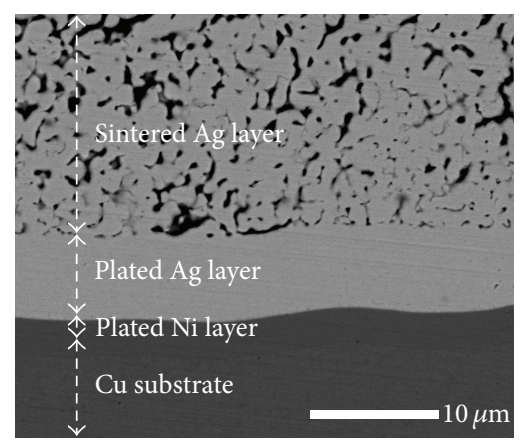

(b)

FIgURE 6: SEM cross-sectional images of joint bonded using Ag nanoparticles at temperature of $250^{\circ} \mathrm{C}$ under $5 \mathrm{MPa}$.

may be related to the vaporization and decomposition of organic component in the Ag nanoparticle paste. Although the $\mathrm{Ag}$ content is increased to 96.1 mass\%, there is still approximately 3.9 mass\% of organic components in the $\mathrm{Ag}$ nanoparticle paste. The decrease of joint strength at $10 \mathrm{MPa}$ may be due to the residue of organic in the sintered Ag layer.

Figure 6 shows SEM cross-sectional images of joint bonded using $\mathrm{Ag}$ nanoparticles at temperature of $250^{\circ} \mathrm{C}$ under $5 \mathrm{MPa}$. The plated Ni layer is introduced to increase the combination between the plated $\mathrm{Ag}$ layer and $\mathrm{Cu}$ substrate during the electrical plating. It can be seen that interconnected microstructures are formed in the sintered Ag layer. There are no obvious defects existing between the interface of different layers, which indicates the good bonding quality of the joint. Figure 7 shows SEM images of the fracture surface of a joint made using $\mathrm{Ag}$ nanoparticles at big $\mathrm{Cu}$ disc and small $\mathrm{Cu}$ disc. Figure 8 presents SEM fracture surfaces images at different areas shown by arrows in Figure 7. It can be seen that fracture traces existed at different areas in the big $\mathrm{Cu}$ disc and small disc. This suggests that the fracture of the joint occurs in the sintered $\mathrm{Ag}$ layer instead of the interface between sintered Ag layer and plated Ag layer.

3.3. High Temperature Properties of the Joint with Ag Nanoparticle Paste. Figure 9 illustrates the shear strength of the joints bonded using $\mathrm{Ag}$ nanoparticle paste and Pb95Sn5 solder after heat treatment at different temperatures from $200^{\circ} \mathrm{C}$ to $350^{\circ} \mathrm{C}$ for 50 hours. Before this heat treatment, the shear strengths of the joints bonded using Ag nanoparticle paste and $\mathrm{Pb} 95 \mathrm{Sn} 5$ solder are $36 \mathrm{MPa}$ and $30 \mathrm{MPa}$, respectively. 


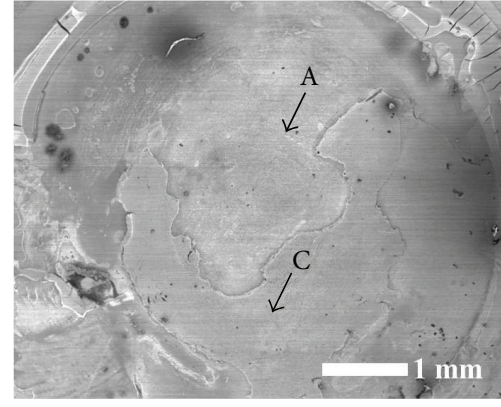

(a)

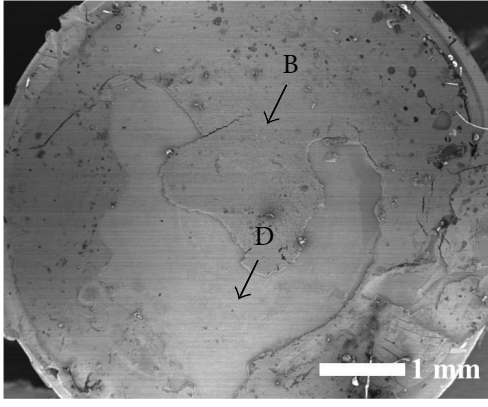

(b)

FIGURE 7: SEM images of fracture surface of joint using Ag nanoparticles: big Cu disc (a); small Cu disc (b).

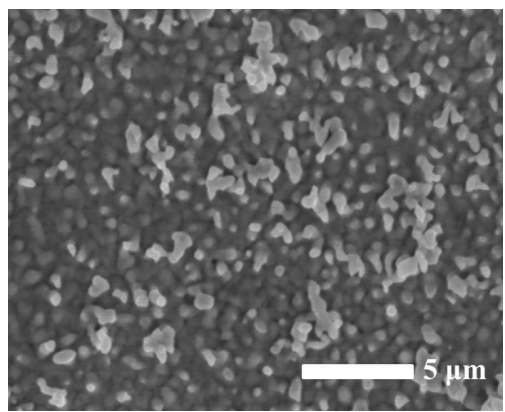

(a)

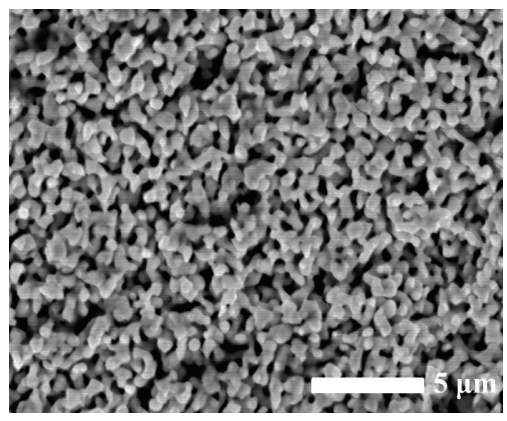

(c)

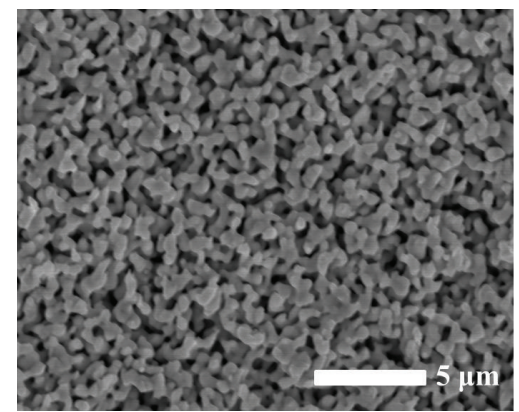

(b)

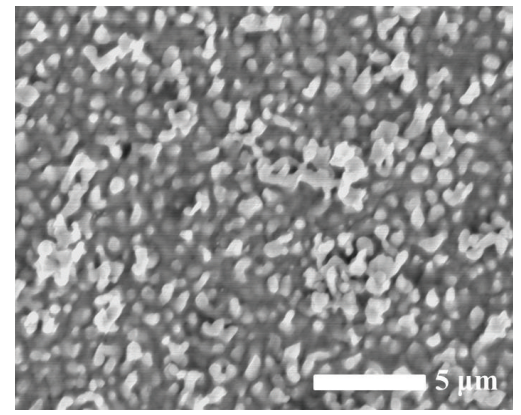

(d)

FIGURE 8: SEM fracture surface images at different areas shown by arrows in Figure 7: area A (a); area B (b); area C (c); area D (d).

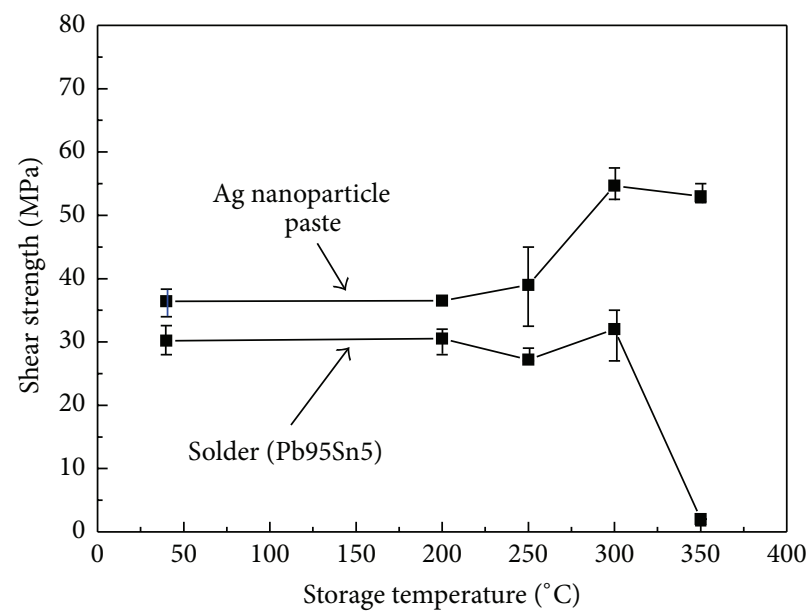

FIGURE 9: Comparison of the joint strength bonded with Ag nanoparticle paste and solder after storage at different temperatures for 50 hours. 


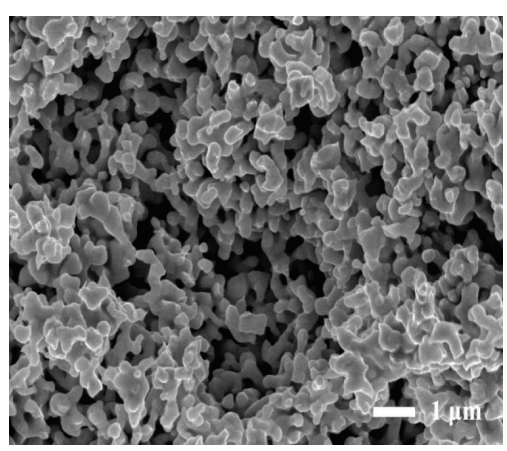

(a)



(c)

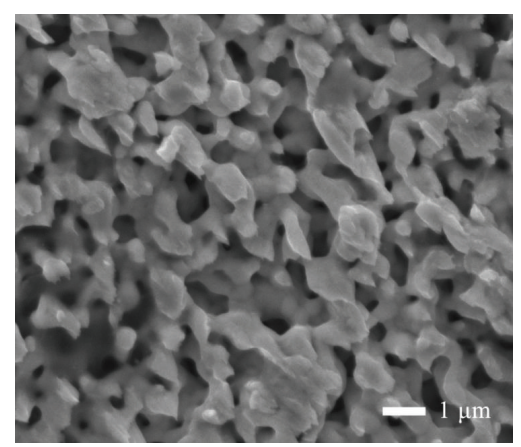

(b)

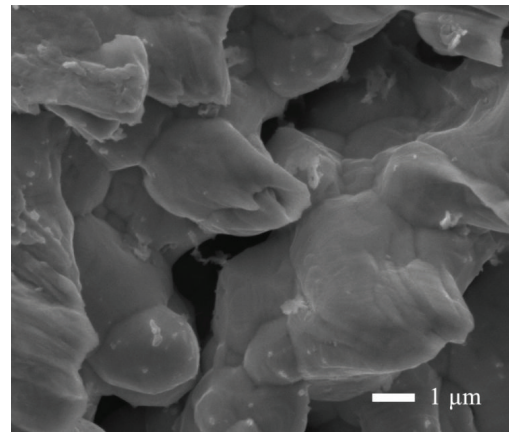

(d)

FIGURE 10: SEM fracture surfaces of the joints bonded using silver nanoparticle paste after heat treatment at different temperatures for 50

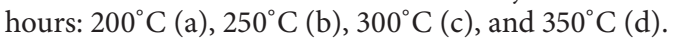

The shear strength of joints bonded using Ag nanoparticle paste increases after heat treatment at temperature range from $200^{\circ} \mathrm{C}$ to $300^{\circ} \mathrm{C}$. After heat treatment at $350^{\circ} \mathrm{C}$ for 50 hours, the joint had a high strength of $50 \mathrm{MPa}$. The strengthening of the joints during heat treatment is due to the further sintering of Ag nanoparticle paste. Figure 10 shows the corresponding fracture microstructures of the joints bonded using silver nanoparticle paste after heat treatment at temperatures. It can be seen that both neck growth and grain growth are detected. It has been shown that the shear strength with Ag nanoparticle paste increases with the growth of neck size; it is reasonable to expect that the strength increases after storage at high temperatures. For the joint bonded with solder Pb95Sn5, no obvious changes of the joint strength were observed after heat treatment from $200^{\circ} \mathrm{C}$ to $300^{\circ} \mathrm{C}$ as shown in Figure 9. After heat treatment at $350^{\circ} \mathrm{C}$ for 50 hours, the joint bonded with solder Pb95Sn5 disconnected and the joint strength decreased to $0 \mathrm{MPa}$ as shown in Figure 9. Figure 11 shows the SEM fracture surfaces of the joints bonded using solder Pb95Sn5 after heat treatment at different temperatures. For the joints after heat treatment at temperatures from $200^{\circ} \mathrm{C}$ to $300^{\circ} \mathrm{C}$, the solder layer with dimple fracture microstructures can be seen (Figures 11(a), 11(b), and 11(c)). Figure 11(d) shows the disconnected surface of the joint bonded with solder Pb95Sn5 after heat treatment at $350^{\circ} \mathrm{C}$. Figure 12 is the EDS analysis result on the surface of disconnected joint. Besides the $\mathrm{Sn}$ and $\mathrm{Ag}$ elements, $\mathrm{Cu}$ and $\mathrm{O}$ were also detected. This suggests that the plated $\mathrm{Ni} / \mathrm{Ag}$ layer on $\mathrm{Cu}$ substrate is broken and oxidation occurred. This is due to the fact that the heat treatment temperature $\left(350^{\circ} \mathrm{C}\right)$ is higher than the melting temperature of solder Pb95Sn5 $\left(300 \sim 314^{\circ} \mathrm{C}\right)$. From these results, it is known that the joints bonded using Ag nanoparticle paste have similar or better mechanical properties compared with the joint bonded with solder Pb95Sn5 in high temperature environments.

\section{Conclusions}

The sintering bonding processes with Ag nanoparticle paste were performed at different bonding pressures from $0 \mathrm{MPa}$ to $10 \mathrm{MPa}$. The increase in bonding pressure from $0 \mathrm{MPa}$ to $7.5 \mathrm{MPa}$ yields stronger joints, which is attributed to the neck growth in sintered Ag layers. The strength of joints bonded at $10 \mathrm{MPa}$ is lower than that bonded at $7.5 \mathrm{MPa}$. This may be due to the residue of organic components in the sintered $\mathrm{Ag}$ layer. The joint properties in high temperature environment are evaluated by the heat treatment at temperatures ranges of $200-350^{\circ} \mathrm{C}$ for 50 hours. For the joints bonded with $\mathrm{Ag}$ nanoparticle paste, the strength increases after heat treatment. This is attributed to the further sintering and neck growth of Ag nanoparticles. For the joints bonded with solder $\mathrm{Pb} 95 \mathrm{Sn} 5$, there is no obvious change for the strength after heat treatment at temperature ranges of $200^{\circ} \mathrm{C}$ to $300^{\circ} \mathrm{C}$. After heat treatment at $350^{\circ} \mathrm{C}$, the joint strengths decrease to $0 \mathrm{MPa}$ due to the disconnection and oxidation of $\mathrm{Cu}$ discs. These results show that the Ag nanoparticle paste has potential applications as bonding materials used in high temperature environments. 


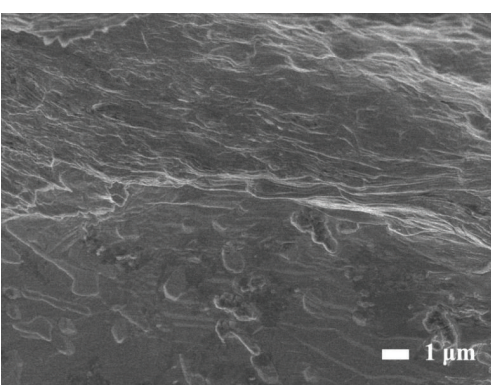

(a)

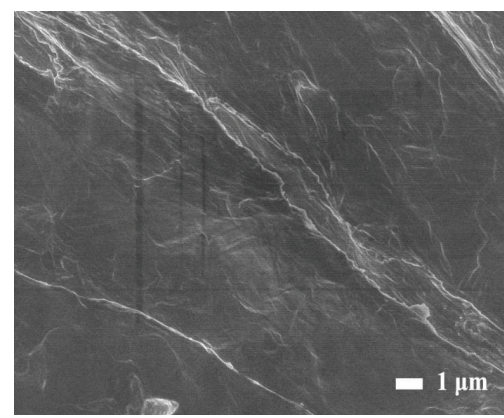

(c)

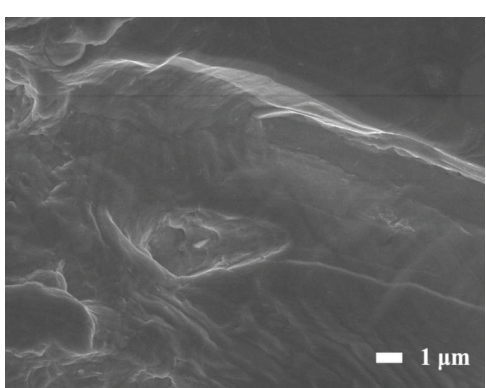

(b)

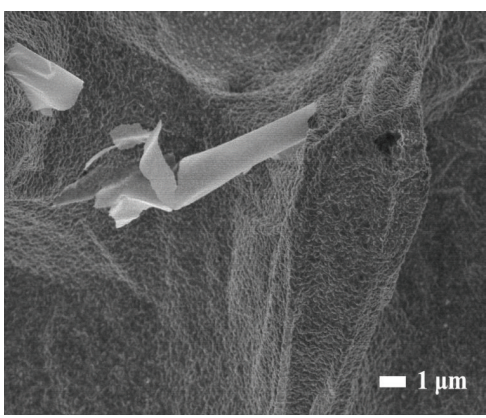

(d)

Figure 11: SEM fracture surfaces of the joints bonded using solder Pb95Sn5 after heat treatment at different temperatures for 50 hours: $200^{\circ} \mathrm{C}$ (a), $250^{\circ} \mathrm{C}(\mathrm{b}), 300^{\circ} \mathrm{C}(\mathrm{c})$, and $350^{\circ} \mathrm{C}(\mathrm{d})$.

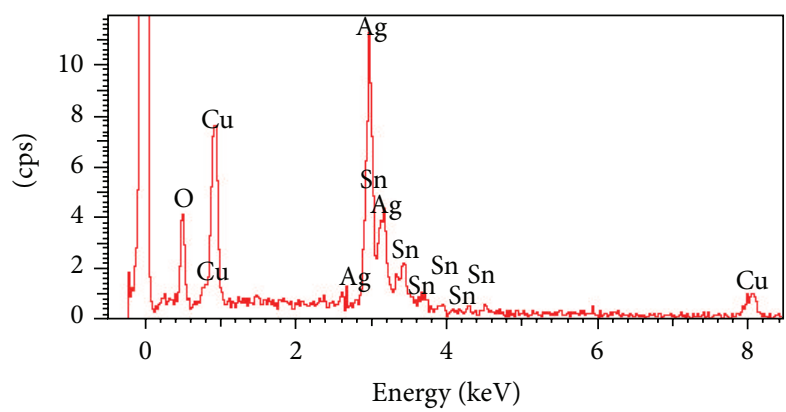

FIGURE 12: EDS analysis on surface of disconnected joint bonded with $\mathrm{Pb} 95 \mathrm{Sn} 5$ after storage at $350^{\circ} \mathrm{C}$ for 50 hours.

\section{Conflict of Interests}

The authors declare that there is no conflict of interests regarding the publication of this paper.

\section{Acknowledgments}

This research was supported by National Science Foundation of China (Grant nos. 51520105007, 51375261, and 51405258), the Natural Science Foundation of Beijing (Grant no. 3132020), The Specialized Research Fund for Doctoral Program of Higher Education (Grant no. 20130002110009), Tsinghua University Initiative Scientific Research Program (Grant nos. 2013THZ02-1, 2013Z02-1), and State Key Laboratory of Automotive Safety and Energy, Tsinghua University (Grant no. 2013XC-B-02).

\section{References}

[1] Y. Li, K.-S. Moon, and C. P. Wong, "Electronics without lead," Science, vol. 308, no. 5727, pp. 1419-1420, 2005.

[2] Q. Jiang, S. H. Zhang, and J. C. Li, "Grain size-dependent diffusion activation energy in nanomaterials," Solid State Communications, vol. 130, no. 9, pp. 581-584, 2004.

[3] P. Buffat and J.-P. Borel, "Size effect on the melting temperature of gold particles," Physical Review A, vol. 13, no. 6, pp. 22872298, 1976.

[4] K.-S. Moon, H. Dong, R. Maric et al., "Thermal behavior of silver nanoparticles for low-temperature interconnect applications," Journal of Electronic Materials, vol. 34, no. 2, pp. 168-175, 2005.

[5] M. Zhang, M. Y. Efremov, F. Schiettekatte et al., "Size-dependent melting point depression of nanostructures: nanocalorimetric measurements," Physical Review B-Condensed Matter and Materials Physics, vol. 62, no. 15, article 10548, 2000.

[6] Y. Zhou, Microjoining \& Nanojoining, Woodhead, England, UK, CRC Press, 2008.

[7] T. G. Lei, J. N. Calata, G.-Q. Lu, X. Chen, and S. Luo, "Lowtemperature sintering of nanoscale silver paste for attaching large-area $\left(>100 \mathrm{~mm}^{2}\right)$ chips," IEEE Transactions on Components and Packaging Technologies, vol. 33, no. 1, pp. 98-104, 2010.

[8] A. Hu, J. Y. Guo, H. Alarifi et al., "Low temperature sintering of Ag nanoparticles for flexible electronics packaging," Applied Physics Letters, vol. 97, Article ID 153117, 2010.

[9] E. Ide, S. Angata, A. Hirose, and K. F. Kobayashi, "Metal-metal bonding process using Ag metallo-organic nanoparticles," Acta Materialia, vol. 53, no. 8, pp. 2385-2393, 2005. 
[10] Y. Jianfeng, Z. Guisheng, H. Anming, and Y. N. Zhou, "Preparation of PVP coated $\mathrm{Cu}$ NPs and the application for lowtemperature bonding," Journal of Materials Chemistry, vol. 21, no. 40, pp. 15981-15986, 2011.

[11] P. Peng, A. Hu, B. Zhao, A. P. Gerlich, and Y. N. Zhou, "Reinforcement of Ag nanoparticle paste with nanowires for low temperature pressureless bonding," Journal of Materials Science, vol. 47, no. 19, pp. 6801-6811, 2012.

[12] Y. Li, D. Li, C. Li et al., "Annealing-induced highly-conductive and stable $\mathrm{Cu}$-organic composite nanoparticles with hierarchical structures," Journal of Alloys and Compounds, vol. 636, pp. $1-7,2015$.

[13] M. Maruyama, R. Matsubayashi, H. Iwakuro, S. Isoda, and T. Komatsu, "Silver nanosintering: a lead-free alternative to soldering," Applied Physics A: Materials Science \& Processing, vol. 93, no. 2, pp. 467-470, 2008.

[14] T. Morita, E. Ide, Y. Yasuda, A. Hirose, and K. Kobayashi, "Study of bonding technology using silver nanoparticles," Japanese Journal of Applied Physics, vol. 47, no. 8, pp. 6615-6622, 2008.

[15] J. G. Bai and L. Guo-Quan, "Thermomechanical reliability of low-temperature sintered silver die attached $\mathrm{SiC}$ power device assembly," IEEE Transactions on Device and Materials Reliability, vol. 6, no. 3, pp. 436-441, 2006.

[16] Y. Akada, H. Tatsumi, T. Yamaguchi, A. Hirose, T. Morita, and E. Ide, "Interfacial bonding mechanism using silver metalloorganic nanoparticles to bulk metals and observation of sintering behavior," Materials Transactions, vol. 49, no. 7, pp. 15371545, 2008.

[17] J. Yan, G. Zou, A. Wu et al., "Effect of PVP on the low temperature bonding process using polyol prepared Ag nanoparticle paste for electronic packaging application," Journal of Physics: Conference Series, vol. 379, no. 1, Article ID 012024, 2012.

[18] J. Yan, G. Zou, A.-P. Wu et al., "Pressureless bonding process using Ag nanoparticle paste for flexible electronics packaging," Scripta Materialia, vol. 66, no. 8, pp. 582-585, 2012.

[19] J. Yan, G. Zou, A. Wu, J. Ren, A. Hu, and Y. N. Zhou, "Improvement of bondability by depressing the inhomogeneous distribution of nanoparticles in a sintering bonding process with silver nanoparticles," Journal of Electronic Materials, vol. 41, no. 7, pp. 1924-1930, 2012.

[20] J. Yan, G. Zou, L. Liu et al., "Sintering mechanisms and mechanical properties of joints bonded using silver nanoparticles for electronic packaging applications," Welding in the World, vol. 59, no. 3, pp. 427-432, 2015.

[21] Y. Sun and Y. Xia, "Shape-controlled synthesis of gold and silver nanoparticles," Science, vol. 298, no. 5601, pp. 2176-2179, 2002.

[22] M. Kerker, "The optics of colloidal silver: something old and something new," Journal of Colloid and Interface Science, vol. 105, no. 2, pp. 297-314, 1985.

[23] H. Wang, X. Qiao, J. Chen, X. Wang, and S. Ding, "Mechanisms of PVP in the preparation of silver nanoparticles," Materials Chemistry and Physics, vol. 94, no. 2-3, pp. 449-453, 2005.

[24] C. Herring, "Some theorems on the free energies of crystal surfaces," Physical Review, vol. 82, no. 1, article 87, 1951. 

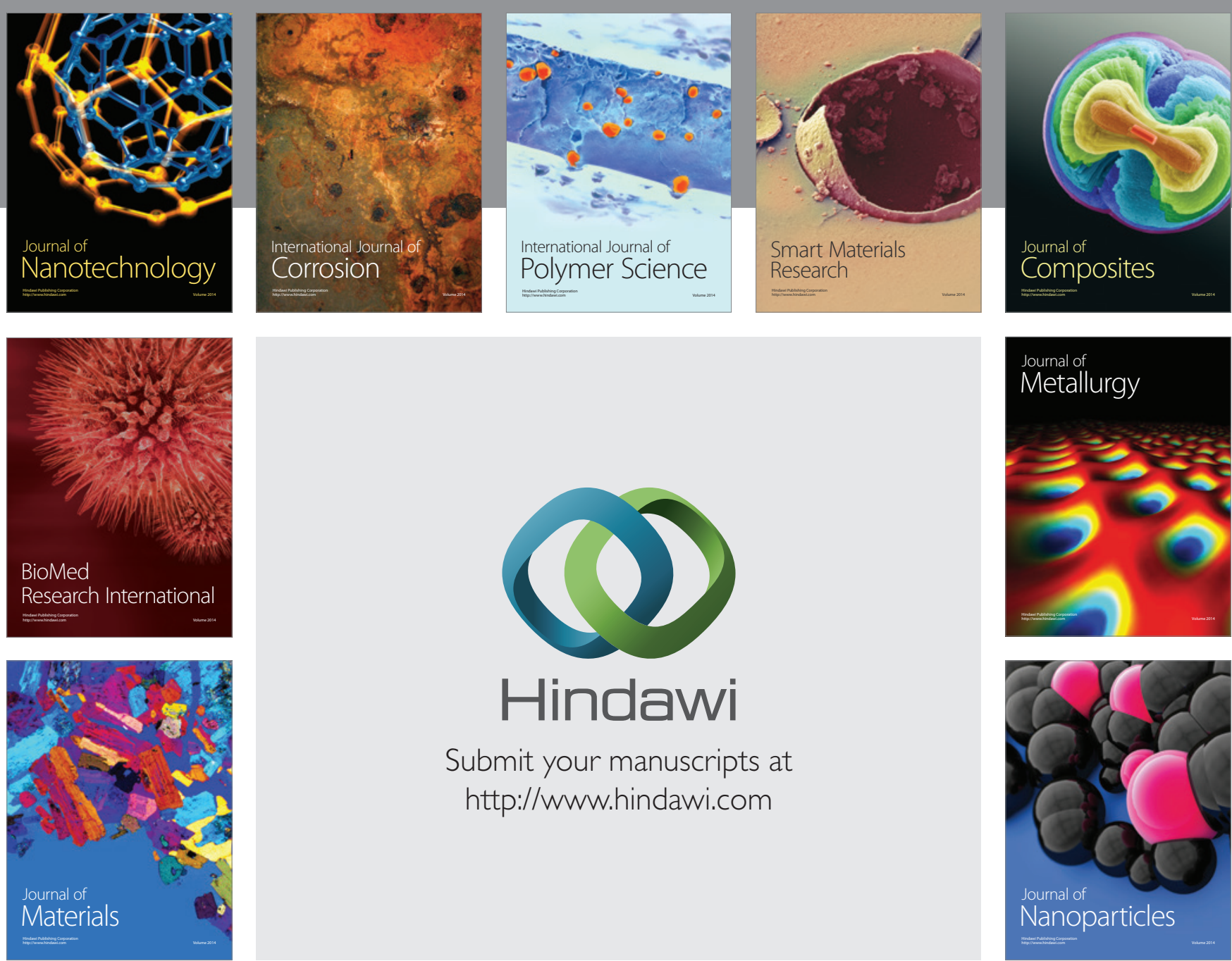

\section{Hindawi}

Submit your manuscripts at

http://www.hindawi.com

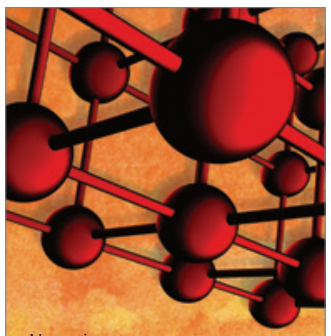

Materials Science and Engineering
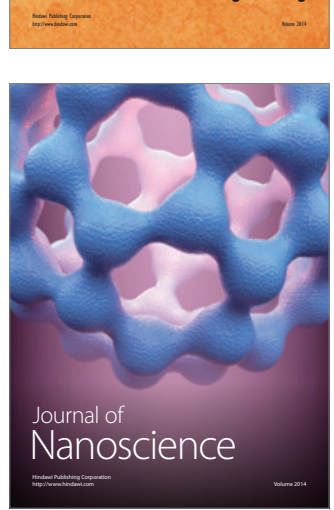
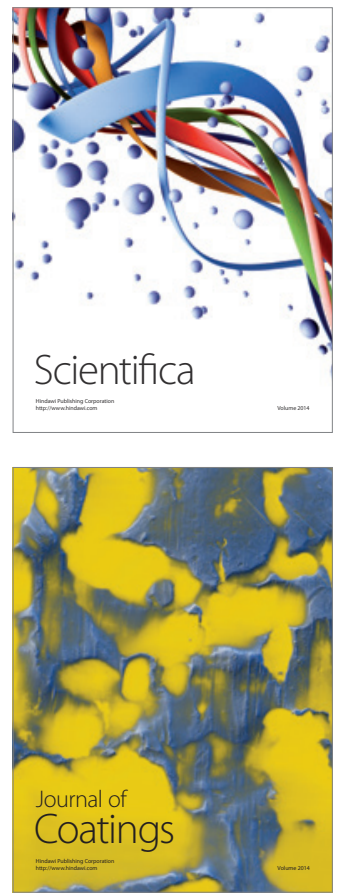
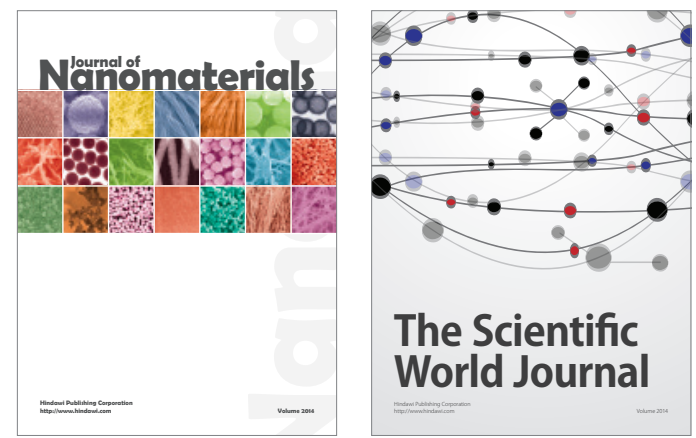

The Scientific World Journal
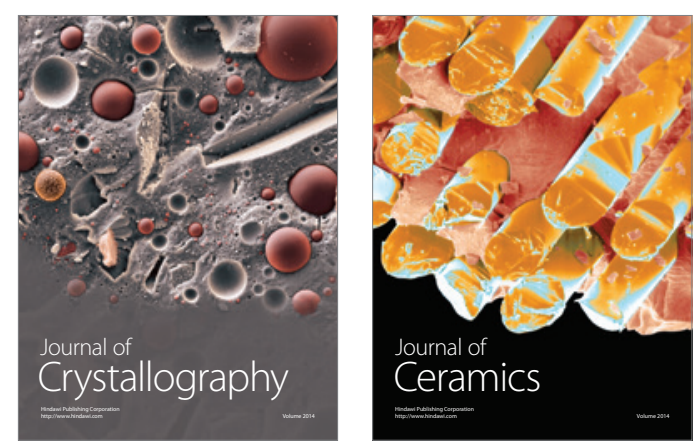
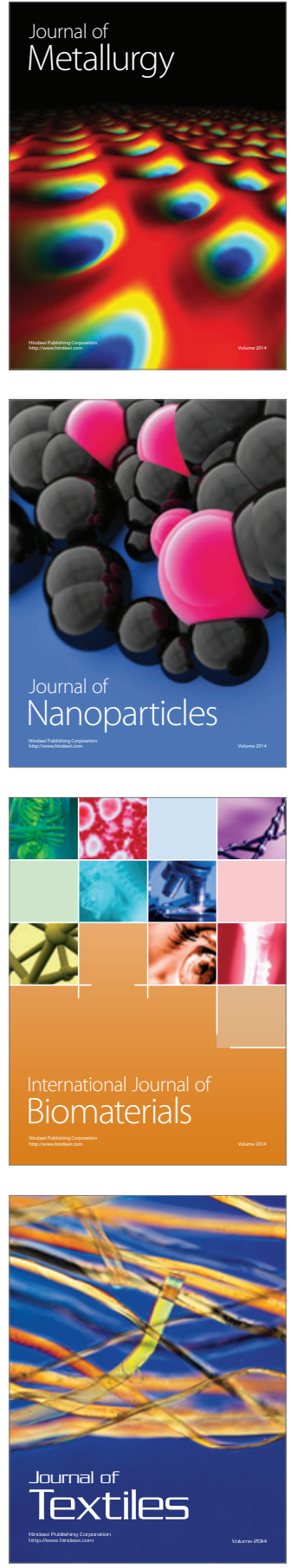